\title{
Organic Light-Emitting Diodes with Multi-Periodic Extraction Gratings
}

\author{
Matthias Bremer, Nicolai Beck, Hannes Lüder, and Martina Gerken \\ Institute of Electrical and Information Engineering, Christian-Albrechts-Universität zu Kiel, Kaiserstr. 2, 24143 Kiel, Germany \\ *mab@tf.uni-kiel.de
}

\begin{abstract}
OLEDs with integrated multi-periodic gratings were fabricated and compared to finite-difference time-domain (FDTD) simulations. Potentially such OLEDs may be used as integrated light sources with tailored emission for microsystems.

OCIS codes: (050.5298) Photonic crystals; (160.4236) Nanomaterials; (230.3670) Light-emitting diodes, (260.5740) Resonance
\end{abstract}

\section{Introduction}

Organic light emitting diodes (OLEDs) are promising as integrated light sources for microsystems as they may be fabricated on a variety of different substrates [1-4]. In microsystems directed emission characteristics rather than a Lambertian emission profile are desired. While organic lasers offer highly directed emission characteristics, so far an additional pump source is necessary [5]. As an alternative, nanostructures may be integrated into OLEDs for tailoring the emission profile. We previously investigated the tailoring of photoluminescence characteristics with multi-periodic gratings constructed by a logical disjunction superposition of binary single-periodic gratings with different grating pitches [6]. The resulting structures possess a grating period that is the least common multiple of the individual grating periods they consist of. Emission peaks in angle and wavelength as well as the relative strengths of the peaks are designed by choosing appropriate grating period and duty cycle combinations [7]. Here, we demonstrate for the first time electroluminescence of an OLED with an integrated multi-periodic grating.

\section{Fabrication of OLEDs with integrated multi-periodic gratings}

In order to fabricate $400 \mu \mathrm{m}$ by $400 \mu \mathrm{m}$ nanostructured OLEDs we developed a lithography-based fabrication process. Fig. 1 shows the schematic of the resulting OLED layer stack. We investigated nanostructures with the following period combinations (the first two are monoperiodic, for the other structures the different periods are separated by slashes): $350 \mathrm{~nm} ; 450 \mathrm{~nm} ; 350 \mathrm{~nm} / 450 \mathrm{~nm} ; 350 \mathrm{~nm} / 400 \mathrm{~nm} / 450 \mathrm{~nm} ; 300 \mathrm{~nm} / 400 \mathrm{~nm} / 500 \mathrm{~nm}$.

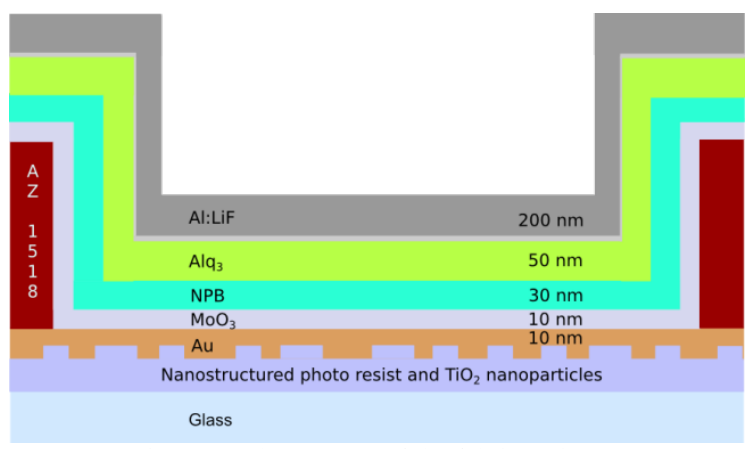

Fig. 1. Stack structure of the fabricated OLEDs.

In the first fabrication step the $500 \mu \mathrm{m}$ by $500 \mu \mathrm{m}$ nanostructure is replicated from an electron-beam written master stamp into the UV curable nanoimprint resist AMONIL (Amo $\mathrm{GmbH}$ ) on a glass substrate. The nanoimprint resist is blended with $\mathrm{TiO}_{2}$ nanoparticles (dispersed in xylene, Sigma-Aldrich Co. LLC) in order to tailor its refractive index [8]. The resist-nanoparticle layer is cured by UV exposure. It has a thickness of $515 \mathrm{~nm}$ and a structure depth of approximately $60 \mathrm{~nm}$. A scanning electron microscopy (SEM) image of a resulting structure is presented in Fig. 2 on the right-hand side.

In the second fabrication step the OLED anode is fabricated. A 10-nm gold layer and a 10-nm $\mathrm{MoO}_{3}$ layer are deposited as a stable, semitransparent anode. For the anode, a large-area shadow mask is employed. 


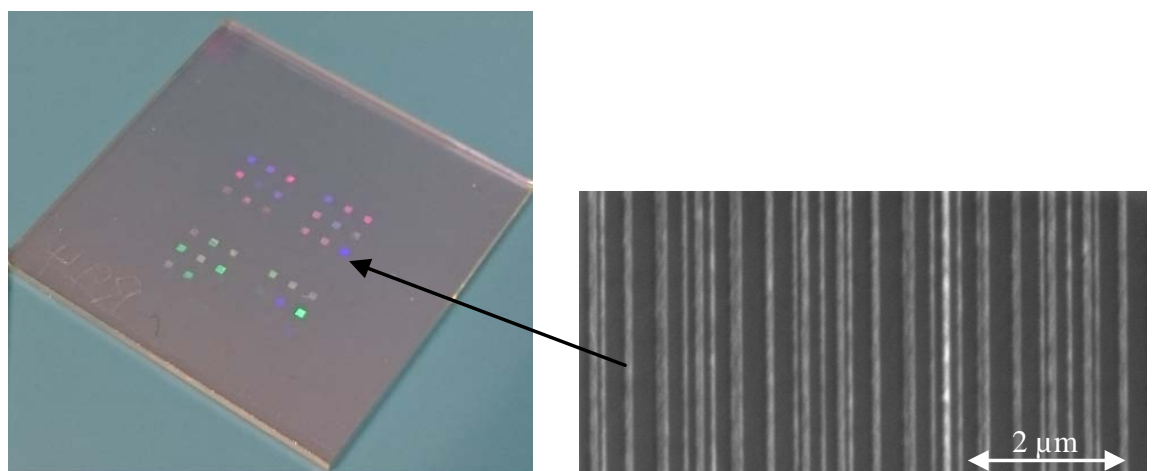

Fig. 2. Left: Photo of replicated multi-periodic gratings in photo resist with $\mathrm{TiO}_{2}$ particles. Right: SEM image of a grating.

In the third fabrication step the $400 \mu \mathrm{m}$ by $400 \mu \mathrm{m}$ size of the OLED is defined by an insulation layer between anode and cathode. Here, we use a $2 \mu \mathrm{m}$ layer of the photo resist AZ1518 (Microchemicals) and structure it in a lithography step.

In the final forth fabrication step the organic layers and the cathode are deposited. For a simple OLED we thermally evaporate N,N'-Di-[(1-naphthyl)-N,N'-diphenyl]-1,1'-biphenyl)-4,4'-diamine (NPB), Tris-(8hydroxychinolin) aluminum $\left(\mathrm{Alq}_{3}\right)$, lithium fluoride and aluminum. For testing the OLEDs are encapsulated with another glass plate and epoxy adhesive.

\section{Comparison of photo- and electroluminescence}

Photoluminescence measurements are helpful to understand the mechanism of guided mode extraction from organic layers. Therefore, we sputter $70 \mathrm{~nm} \mathrm{TiO}{ }_{2}$ on a simple stamped Amonil layer with a thickness of $200 \mathrm{~nm}$ and evaporate a layer of $50 \mathrm{~nm} \mathrm{Alq}$ plus $100 \mathrm{~nm} \mathrm{MoO}$ as encapsulation layer on top. The produced sample is characterized in a goniophotometer in combination with a spectrometer (Andor Shamrock SR-500i) with a cooled back-illuminated CCD detector (Andor DU920P-OE) at $-60^{\circ} \mathrm{C}$ and $1 \mathrm{~s}$ integration time. A structured area is then excited with a diode laser of $405 \mathrm{~nm}$ wavelength. The angle-resolved photoluminescence spectrum is shown in Fig. 3 on the right. The resonances caused by the multi-periodic grating are clearly visible.

We simulated the device behavior with the finite-difference time-domain (FDTD) method employing the software FDTD Solutions (Lumerical Solutions Inc.). Material losses and dispersion are included in the twodimensional model. Modal excitation with the fundamental TE and TM modes is used and the spectra are weighted by the $\mathrm{Alq}_{3}$ spectrum. Figure 3 presents the superposition of the TE and TM simulation results on the left-hand side. In this figure no background radiation is visible as only waveguide mode scattering is considered. The resonances are shifted compared to the measurements most likely due to a parameter deviation in the experiment.
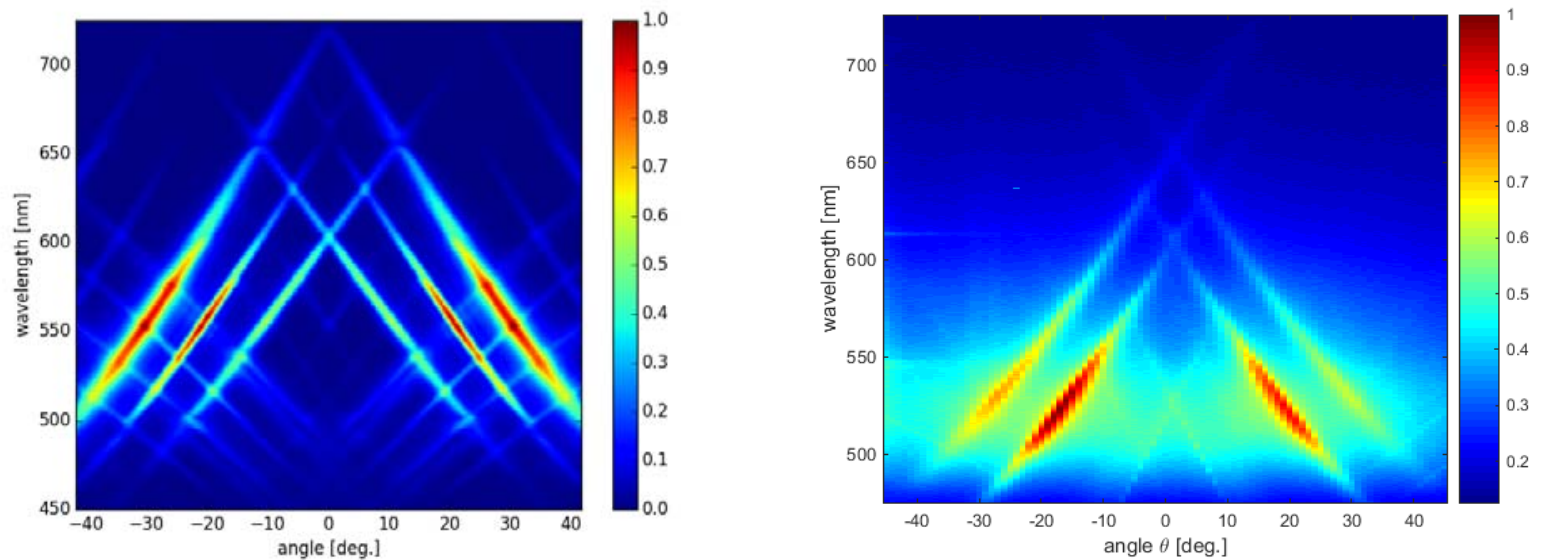

Fig. 3. Angle-resolved photoluminescence spectra of an $\mathrm{Alq}_{3}$ layer on a photonic crystal with two superimposed grating periods of $350 \mathrm{~nm}$ and $450 \mathrm{~nm}$; Left: FDTD simulation of waveguide mode scattering; Right: Measurement.

The simulation for the electroluminescence of the complete OLED is given in Fig. 3 on the left and the measurement next to it on the right. The integration time was $10 \mathrm{~s}$ in this case. Resonances of the gratings are still visible in the FDTD simulation though the absorption by the included electrodes is quite high. The resonances in 
the goniometric measurements are hardly visible. Additionally, interference effects are observed due to reflections into the cathode-insulation layer-anode Fabry-Perot stack.
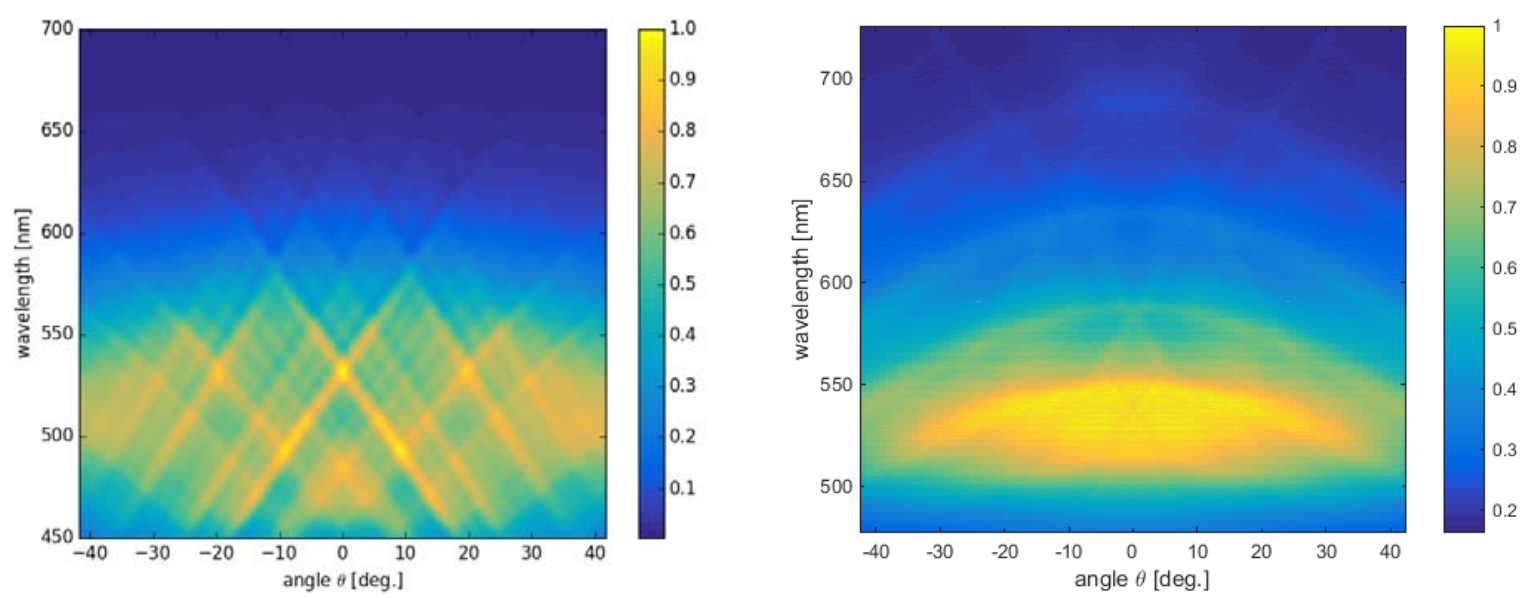

Fig. 4. Angle-resolved electroluminescence spectra of an OLED with two superimposed grating periods of $350 \mathrm{~nm}$ and $450 \mathrm{~nm}$; Left: FDTD simulation; Right: Measurement.

\section{Conclusion}

$400 \mu \mathrm{m}$ by $400 \mu \mathrm{m}$ OLEDs with integrated multi-periodic nanostructures were fabricated successfully implementing a cost-efficient UV nanoimprint process. Electroluminescence of multi-periodic OLEDs was demonstrated successfully for the first time. Unfortunately, the observed grating resonances in the spectrum are weak. We attribute this to the short absorption length compared to the grating scattering length in the OLED waveguide. Therefore, we aim to reduce losses by reducing absorption. For example, indium tin oxide (ITO) may be deposited instead of gold as a low-loss anode. As photoluminescence of nanostructured waveguides shows high intensity enhancement ratios of up to 40:1 compared to the background [7], we expect much better enhancement factors, when absorption is reduced. Small OLEDs with tailored emission profiles are promising for microsystems such as integrated optical sensors. Using a multi-periodic grating design, it is possible to supply different spatial positions with designed illumination wavelength or angle.

\section{Acknowledgements}

The authors gratefully acknowledge the support by the ERC within the "PhotoSmart" project (Starting Grant Agreement 307800). The authors thank M. Köpke for technical support. This work was carried out with the support of the Karlsruhe Nano and Micro Facility (KNMF, www.kit.edu/knmf), a Helmholtz Research Infrastructure at Karlsruhe Institute of Technology (KIT, www.kit.edu).

[1] J. Liang, L. Li, X. Niu, Z. Yu, and Q. Pei, “Elastomeric polymer light-emitting devices and displays,” Nat. Photonics 7, 817-824 (2013).

[2]Z. B. Wang, M. G. Helander, J. Qiu, D. P. Puzzo, M. T. Greiner, Z. M. Hudson, S. Wang, Z. W. Liu, and Z. H. Lu, "Unlocking the full potential of organic light-emitting diodes on flexible plastic,” Nat. Photonics 5, 753-757 (2011).

[3] M. S. White, M. Kaltenbrunner, E. D. Głowacki, K. Gutnichenko, G. Kettlgruber, I. Graz, S. Aazou, C. Ulbricht, D. a. M. Egbe, M. C. Miron, Z. Major, M. C. Scharber, T. Sekitani, T. Someya, S. Bauer, and N. S. Sariciftci, "Ultrathin, highly flexible and stretchable PLEDs," Nat. Photonics 7, 811-816 (2013).

[4] W. H. Koo, S. M. Jeong, F. Araoka, K. Ishikawa, S. Nishimura, T. Toyooka, and H. Takezoe, "Light extraction from organic light-emitting diodes enhanced by spontaneously formed buckles,” Nat. Photonics 4, 222-226 (2010).

[5] M. T. Hill, and M. C. Gather, “Advances in small lasers,” Nat. Photonics 8, 908-918 (2014).

[6] C. Kluge, M. Rädler, A. Pradana, M. Bremer, P.-J. Jakobs, N. Barié, M. Guttmann, and M. Gerken, "Extraction of guided modes from organic emission layers by compound binary gratings,” Opt. Lett. 37, 2646-8 (2012).

[7] C. Kluge, J. Adam, N. Barié, P.-J. Jakobs, M. Guttmann, and M. Gerken, “Multi-periodic nanostructures for photon control,” Opt. Express 22, A1363 (2014).

[8] A. Pradana, C. Kluge, and M. Gerken, “Tailoring the refractive index of nanoimprint resist by blending with TiO_2 nanoparticles,” Opt. Mater. Express 4, 329 (2014). 